\title{
Solar Irradiance Variability Due to Solar Flares Observed in Lyman-Alpha Emission
}

\author{
Ryan O. Milligan ${ }^{1}$
}

Received: 27 November 2020 / Accepted: 25 February 2021 / Published online: 18 March 2021

(C) The Author(s) 2021

\begin{abstract}
As the Lyman-alpha $(\operatorname{Ly} \alpha)$ line of neutral hydrogen is the brightest emission line in the solar spectrum, detecting increases in irradiance due to solar flares at this wavelength can be challenging due to the very high background. Previous studies that have focused on the largest flares have shown that even these extreme cases generate enhancements in Ly $\alpha$ of only a few percent above the background. In this study, a superposed-epoch analysis was performed on $\approx 8500$ flares greater than $\mathrm{B} 1$ class to determine the contribution that they make to changes in the solar EUV irradiance. Using the peak of the $1-8 \AA \mathrm{X}$-ray emission as a fiducial time, the corresponding time series of $3123 \mathrm{~B}$ - and $4972 \mathrm{C}$-class flares observed in Ly $\alpha$ emission by the EUV Sensor on the Geostationary Operational Environmental Satellite 15 (GOES-15) were averaged to reduce background fluctuations and improve the flare signal. The summation of these weaker events showed that they produced a $0.1-0.3 \%$ enhancement to the solar Ly $\alpha$ irradiance on average. For comparison, the same technique was applied to $453 \mathrm{M}$ - and $31 \mathrm{X}$-class flares, which resulted in a $1-4 \%$ increase in Ly $\alpha$ emission. Flares were also averaged with respect to their heliographic angle to investigate any potential center-to-limb variation. For each GOES class, the relative enhancement in Ly $\alpha$ at the flare peak was found to diminish for flares that occurred closer to the solar limb due to the opacity of the line and/or foreshortening of the footpoints. One modest event included in the study, a C6.6 flare, exhibited an unusually high increase in Ly $\alpha$ of $7 \%$ that may have been attributed to a failed filament eruption. Increases of this magnitude have hitherto only been associated with a small number of X-class flares.
\end{abstract}

Keywords Solar flares · Solar chromosphere $\cdot$ Lyman-alpha $\cdot$ Solar Irradiance Center-to-limb variation

\footnotetext{
R.O. Milligan

r.milligan@qub.ac.uk

1 Astrophysics Research Centre, School of Mathematics and Physics, Queen's University Belfast, University Road, Belfast, BT7 1NN, Northern Ireland, UK
} 


\section{Introduction}

The Lyman-alpha $(\operatorname{Ly} \alpha)$ line of neutral hydrogen at $1216 \AA$ is the brightest emission line in the solar spectrum. It results from the $2 \mathrm{p}-1 \mathrm{~s}$ transition, and in the quiet-Sun, its formation height ranges from the mid-chromosphere $(\approx 6,000 \mathrm{~K}$; line wings) to the base of the transition region $(\approx 40,000 \mathrm{~K}$; line core). During solar flares, nonthermal electrons deposit their energy at these layers, generating localised heating and ionization at the flare footpoints. Precisely how the Ly $\alpha$ line responds to this injection of energy remains unknown due to the lack of spectrally-resolved measurements of this fundamental line during flares. One exception is a serendipitous observation of the 28 October 2003 X17 flare by Woods et al. (2004). They found that the line core increased by around $20 \%$ while the line wings were enhanced by around factor of two, with the blue wing responding more than the red wing (see also Canfield and van Hoosier, 1980; Brekke et al., 1996; and Milligan and Chamberlin, 2016). However, even in this case the data were recorded with a scanning spectrograph and so different parts of the line profile may have been sampled at different stages of the flare's impulsive phase.

Ly $\alpha$ is believed to be a significant radiator of flare energy, accounting for several percent of the total radiated energy in a single emission line (Rubio da Costa et al., 2009; Milligan et al., 2014). It has also recently been shown that flare-induced acoustic oscillations can be detected in disc-integrated Ly $\alpha$ observations, suggesting a potential diagnostic for the dissipation of mechanical energy as well (Milligan et al., 2017, 2020). The study of flares in Ly $\alpha$ is also crucial for the field of space weather as photons at this wavelength can induce chemical and dynamic changes in planetary atmospheres (Chubb et al., 1957; Lean, 1985; Woods et al., 1995). During quiescent solar conditions, Ly $\alpha$ photons get absorbed in the D-layer of Earth's ionosphere, along with solar X-rays, although Raulin et al. (2013) were unable to detect any appreciable D-layer effects during seven small-to-moderate flares observed in $\operatorname{Ly} \alpha$; these flares were found to produce Ly $\alpha$ enhancements $<1 \%$. However, during an $\mathrm{X}$ class flare that exhibited a $\approx 30 \%$ Ly $\alpha$ enhancement, Milligan et al. (2020) noted that the impulsive increase in $\operatorname{Ly} \alpha$ emission correlated with induced currents in the E-layer due to the ionization of nitric oxide as determined from magnetometer data. The corresponding D-layer response occurred several minutes later in line with the more gradually varying soft $\mathrm{X}$-rays (SXR). Further study of flares in Ly $\alpha$ emission and the corresponding response of the terrestrial atmosphere is required to establish if that was an isolated case, or a more common occurrence.

The few reports of solar flare observations in Ly $\alpha$ that previously existed were mostly from broadband, disc-integrated irradiance measurements, and often focused on small numbers of events due to the limited duty cycles of instruments capable of observing flares at this wavelength (Kretzschmar, Dominique, and Dammasch, 2013; Raulin et al., 2013; Milligan et al., 2014; Kretzschmar, 2015; Milligan and Chamberlin, 2016; Dominique et al., 2018). However, Milligan et al. (2020) recently carried out a statistical study of $477 \mathrm{M}$ - and $\mathrm{X}$-class flares in broadband, disc-integrated Ly $\alpha$ emission, and calculated the distribution of contrasts. It was found that most flares $(95 \%)$ produced less than a $10 \%$ enhancement above background, with a maximum of $\approx 30 \%$, although X-class flares that occurred closer to the solar limb were found to produce less of an enhancement. They also measured how the total energy radiated in Ly $\alpha$ scaled with the energy radiated in X-rays; the excess solar irradiance in Ly $\alpha$ was found to be up to two orders of magnitude more energetic than that of the corresponding X-rays.

The study of Milligan et al. (2020) focused on larger flares as smaller GOES class events (B- and C-class) did not produce an appreciable response in Ly $\alpha$ above the intense background. This may be due to instrumental sensitivity, or perhaps Ly $\alpha$ emission from weaker 
events is not inherently significant. The aim of this article is therefore to use a superposedepoch analysis technique to determine how much of an enhancement these smaller flares would produce, if any, and how much this increase contributes to the solar irradiance at this wavelength. Kretzschmar et al. (2010) used this simple technique to determine the effects of solar flares on the total solar irradiance (TSI; solar flux over all wavelengths incident at the top of Earth's atmosphere). Using the time of peak soft X-ray emission (i.e. the $1-8 \AA$ channel) from the X-Ray Sensor (XRS) on board the Geostationary Operational Environmental Satellite (GOES; Hanser and Sellers, 1996) as a key time marker for several hundred flares, the corresponding TSI time series around each flare could then be coadded (or averaged) to reduce the background fluctuations (due to acoustic oscillations and granulation), thereby increasing the coherent flare signal. Similarly, Kretzschmar (2011) followed this study by using the same technique on visible, EUV, and SXR irradiance datasets from the Solar and Heliospheric Observatory (SOHO) to show that all flares can be considered to be white light flares, and that the visible component of flare emission (corresponding to a temperature of $9,000 \mathrm{~K}$ ) can amount to $70 \%$ of the total radiated energy. Section 2 of this article describes the dataset that was used and how the analysis technique was applied. The results are presented in Section 3, while a summary and discussion are given in Section 4.

\section{Data Analysis}

The Extreme Ultra-Violet Sensor (EUVS; Viereck et al., 2007) on board the GOES-N series of satellites (GOES-13, GOES-14, and GOES-15) comprises five channels, A, B, C, D, and

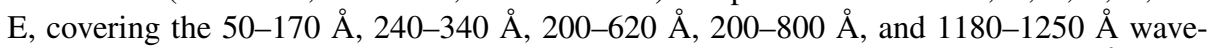
length ranges, respectively, with the E-channel centered on the Ly $\alpha$ line at $1216 \AA$. Each broadband channel samples the solar EUV irradiance at $10.24 \mathrm{~s}$ cadence, compared to the more familiar GOES-N/XRS which samples the 0.5-4 $\AA$ and 1-8 $\AA$ wavelength ranges at 2 s cadence. While the availability of EUVS data from GOES-13 and GOES-14 has been inconsistent since their launches, GOES-15 has provided continuous coverage since its launch in 2010. However, only data taken up until 6 June 2016 have been made publicly available at the time of writing. EUVS-E data are scaled to a Whole Heliosphere Interval quiet-Sun reference spectrum ${ }^{1}$ (Woods et al., 2009), providing Ly $\alpha$ irradiance measurements in physical units of $\mathrm{W} \mathrm{m}^{-2}$. Thus the data may not necessarily reflect flare-related time variations of the line profile, generating some systematic uncertainties. To correct for detector degradation, the EUVS-E data are scaled to the daily-averaged data from the Solar-Stellar Irradiance Comparison Experiment (SOLSTICE; McClintock, Rottman, and Woods, 2005) on board the Solar Radiation and Climate Experiment (SORCE), and only Version 4 of the EUVS-E data were used in this study. ${ }^{2}$

Figure 1 shows a 72-hour period from February 2011, during which, flares of a range of X-ray magnitudes occurred (top panel; the vertical dotted lines in both panels denote the peak times of each X-ray event). However, over the same period, only the largest of these events produced a discernible response in Ly $\alpha$ emission (bottom panel). Also visible in the bottom panel are the daily dips in detected Ly $\alpha$ emission due to attenuation by the Earth's geocorona-which is opaque to Ly $\alpha$ photons (Meier and Prinz, 1970; Baliukin et al., 2019)_for a few hours during each GOES orbit. For every event considered in this study, a 24 hour period of Ly $\alpha$ emission centered on the peak X-ray time of the flare was fitted with

\footnotetext{
${ }^{1}$ http://lasp.colorado.edu/lisird/data/whi_ref_spectra.

${ }^{2}$ https://www.ngdc.noaa.gov/stp/satellite/goes/doc/GOES_NOP_EUV_readme.pdf.
} 


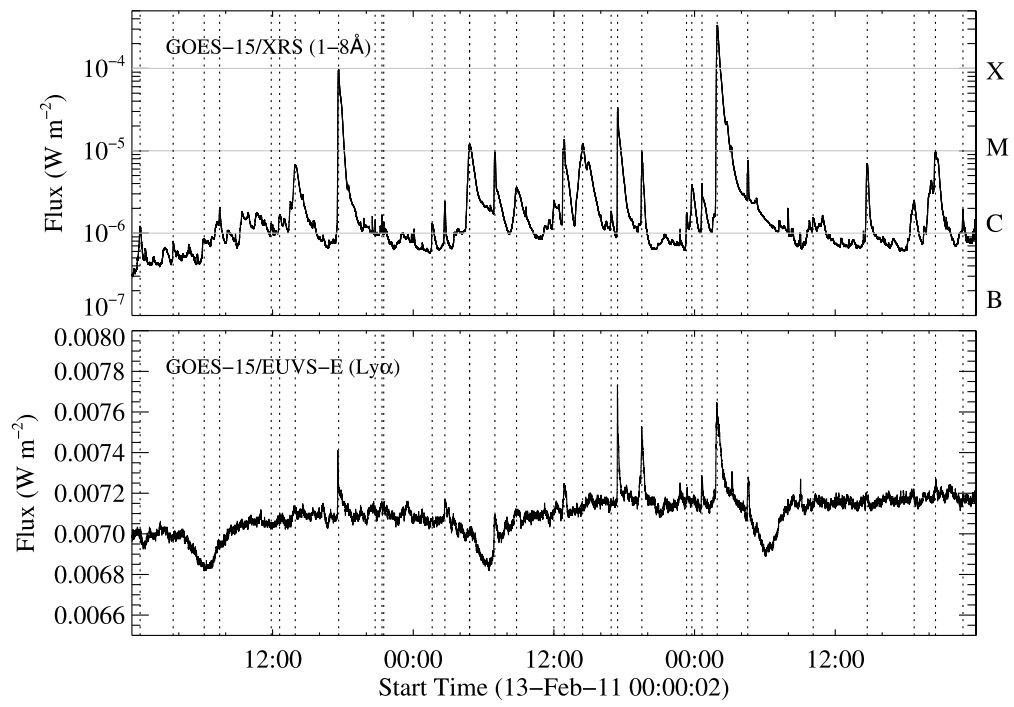

Figure 172 hours of X-ray (top panel) and Ly $\alpha$ (bottom panel) irradiance data from GOES-15 during February 2011. The vertical dotted lines in each panel denote the peak times of all events listed in the NOAA flare catalog. The daily dips in the Ly $\alpha$ data are due to geocoronal absorption by the Earth's outer atmosphere.

a constant, equal to the modal value, plus an inverted Gaussian (red curve in the bottom panel of Figure 2) to account for the geocoronal dip given that the amount of attenuation varies throughout the year. As the effect of the geocorona on Ly $\alpha$ flare irradiance is likely to be nonlinear, any events that occurred within $\pm 2 \sigma$ (vertical dashed line) of the minimum of each daily dip (vertical solid line) were omitted from this study.

The start, peak, and end times of every X-ray flare are listed in the GOES event list ${ }^{3}$ hosted by the National Oceanic and Atmospheric Administration/Space Weather Prediction Center (NOAA/SWPC). However, this list does not always include the flare location, whereas the Heliophysics Event Knowlegebase (HEK) ${ }^{4}$ does, although it is missing several months of data throughout Solar Cycle 24 (Milligan and Ireland, 2018). The superposedepoch technique employed in this study uses the peak time of all X-ray flares above B1 from the HEK database as a fiducial time after removing any events affected by geocoronal absorption, eclipse periods, or corrupted data. This resulted in 3123 B-class and 4972 C-class flares. ${ }^{5}$ While the Ly $\alpha$ component of M- and X-class flares can often be detected above the solar background, the averaged profiles of $453 \mathrm{M}$ - and $31 \mathrm{X}$-class flares were also included in this analysis. This then allows the average flux (in $\mathrm{W} \mathrm{m}^{-2}$ ) and contrast (relative percentage increase) to be measured for each GOES classification (Section 3). Furthermore, given the abundance of events observed by GOES-15/EUVS-E over Solar Cycle 24, and with knowledge of their locations from the HEK, the average Ly $\alpha$ profile can be determined as a function of heliographic angle in order to establish any potential center-to-limb variation (CLV; Section 3.1).

\footnotetext{
${ }^{3}$ https://www.ngdc.noaa.gov/stp/satellite/goes/index.html.

${ }^{4}$ https://www.lmsal.com/hek/.

${ }^{5}$ Note that there were more C-class flares than B-class flares due to the high X-ray background around the time of solar maximum.
} 

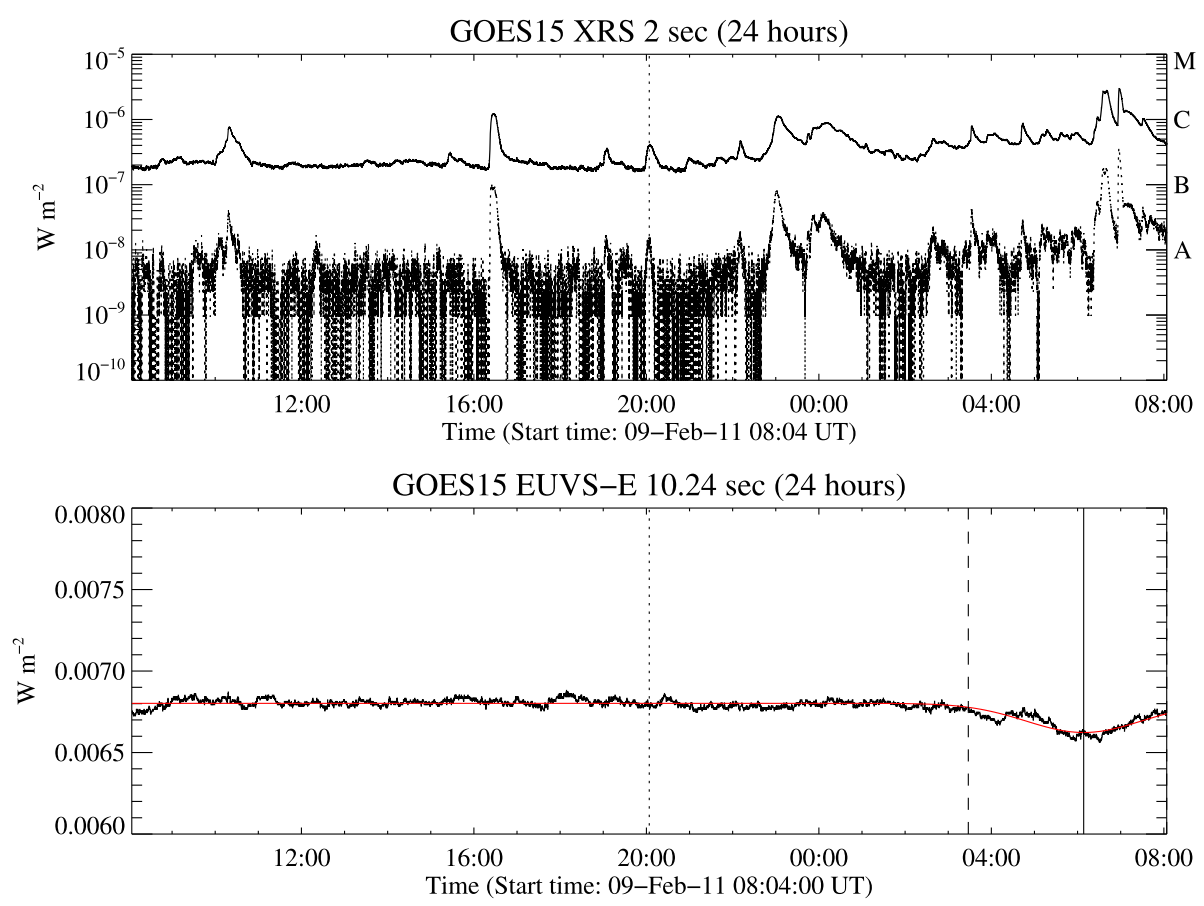

Figure 2 Top panel: 24 hours of GOES-15/XRS data at $1-8 \AA$ (solid curve) and $0.5-4 \AA$ (dotted curve) centered on the peak of the B2.9 flare that occurred on 9 February 2011 (SOL2011-02-09) beginning at 19:57 UT. The vertical dotted line denotes the peak of the B2.9 flare as listed in the NOAA event list. Bottom panel: The GOES-15/EUVS-E (Ly $\alpha$ ) data over the same 24 hour period. The red line marks the background value over this period, taken as a constant equal to the modal value plus a Gaussian to account for geocoronal absorption, seen as a dip in the data around 06:00 UT on 10 February 2011. The vertical solid line marks the minimum of the dip, while the vertical dashed line marks the $2 \sigma$ width.

A one-hour time range of the Ly $\alpha$ time series, from 20 minutes prior to the X-ray peak to 40 minutes after, was averaged over for all flares of a given GOES class. Four B-class flares are shown in the left hand panels of Figure 3 as an example. For each individual event, there does not appear to be any discernible Ly $\alpha$ enhancement due to the flare, but by averaging over increasing numbers of $\operatorname{Ly} \alpha$ time series of equal length, the signal-to-noise ratio is vastly improved as shown in the right hand panel. The red, green, and blue profiles represent the summation of 10, 100, and 1000 randomly chosen B-class flares, respectively. The smoothly-varying solid black lightcurve is the result of averaging all 3123 B-class flares considered. This illustrates how the signal-to-noise ratio is increased by increasing the number of events added together.

\section{Results}

The four panels in Figure 4 show the average Ly $\alpha$ time profiles for B-, C-, M-, and Xclass flares. All profiles show a clear and distinct peak in Ly $\alpha$ emission that was not readily visible for individual events, particularly weaker GOES classes. The average B-class flare produced an enhancement of $0.18 \%$ above background, while C-class flares showed a $0.35 \%$ 

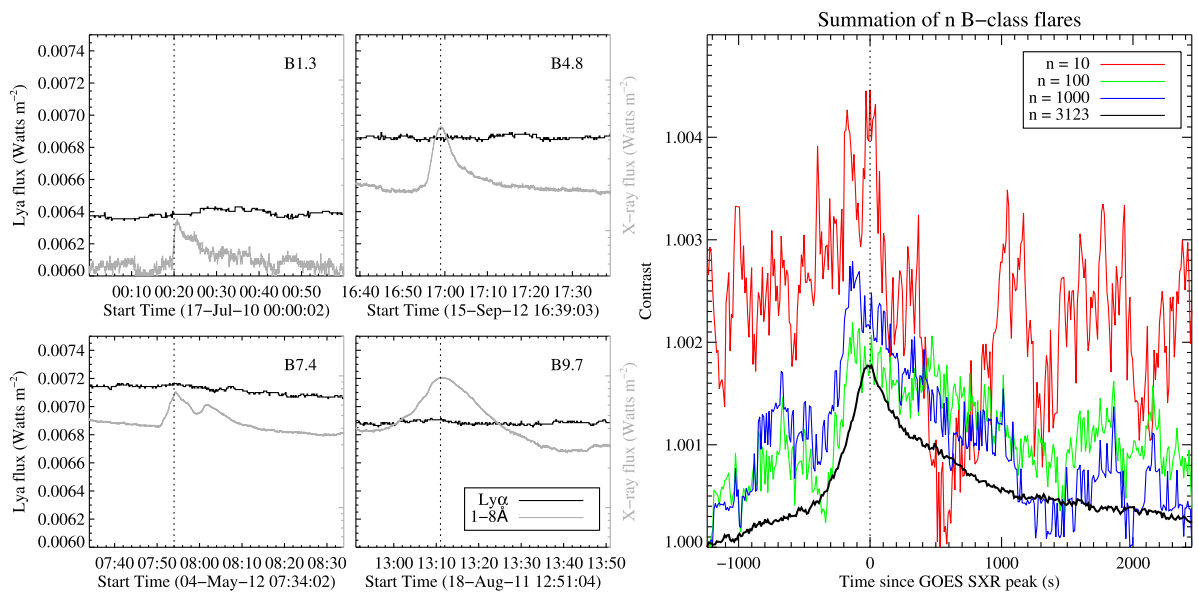

Figure 3 Left hand panels: Time profiles for four B-class flares in Ly $\alpha$ (black) and X-rays (gray). The scaling is the same on each panel. The vertical dotted line on each panel marks the X-ray peak as catalogued in the NOAA flare list. The right hand panel illustrates how the Ly $\alpha$ flare signal becomes more apparent when adding together increasing numbers of events. The red curve is the average of 10 random B-class flares, the green curve is the result of 100 events, the blue curve of 1000, and finally the black curve is an average of all 3123 B-class flares from this study.
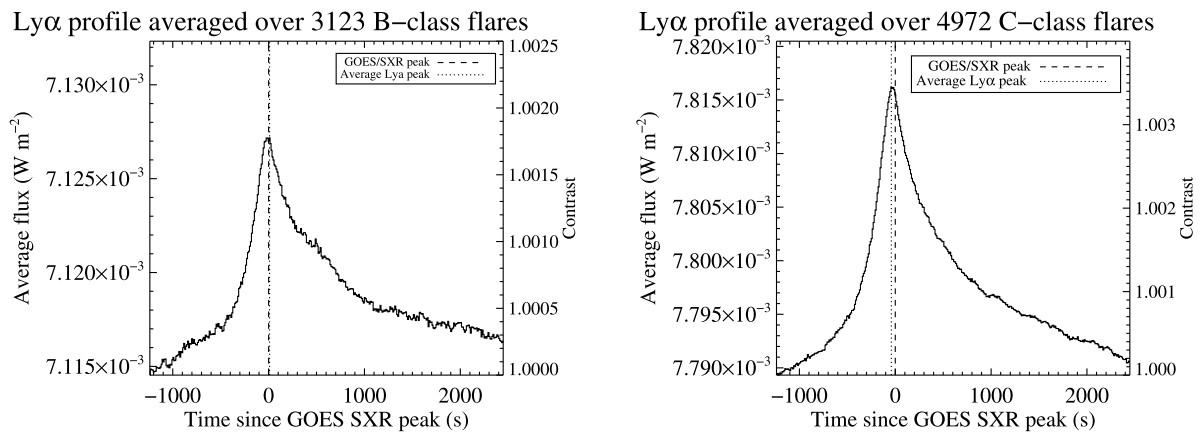

Ly $\alpha$ profile averaged over $453 \mathrm{M}$-class flares
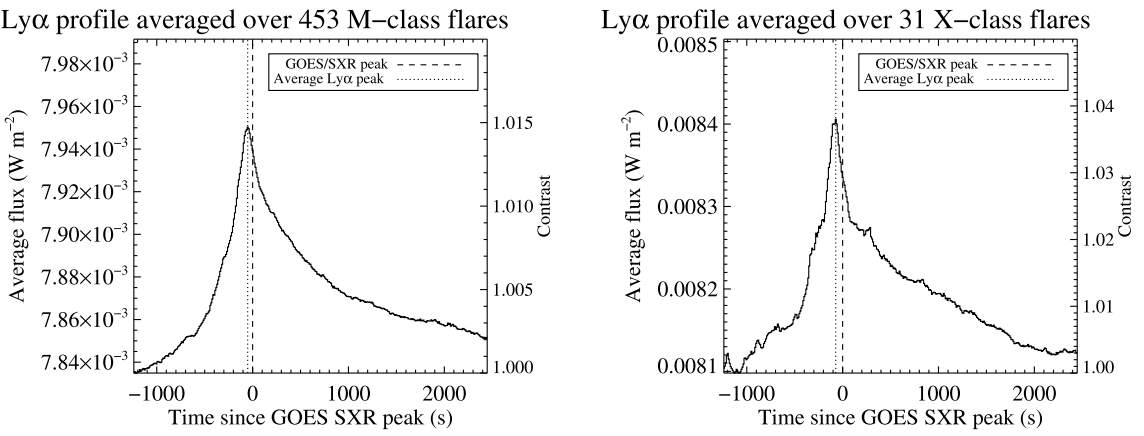

Figure 4 Plots of the average time profiles for all B-, C-, M-, and X-class flares included in this study. The vertical dashed line in each panel marks the time of the SXR peaks of all flares, while the vertical dotted line marks the peak of the Ly $\alpha$ emission. The contrast axis denotes the percentage increase in each panel. 
increase. These values are in agreement with those reported by Raulin et al. (2013) for several C-class flares observed by Project for On-Board Autonomy 2/Large Yield Radiometer (PROBA-2/LYRA). Most individual M- and X-class flares can be readily observed in Ly $\alpha$, as shown by Milligan et al. (2020), they are included here for completeness. M-class flares were measured to increase the solar irradiance by $1.5 \%$ on average, while X-class flares exhibited a 3.8\% increase in Ly $\alpha$ above background. Although the flare-related changes in Ly $\alpha$ irradiance quoted here are quite small, these changes can still correspond to a significant amount of radiated energy. This confirms that weaker flares do have associated responses in Ly $\alpha$ emission that are ordinarily obscured by the intense solar background. This will have significant implications for more sensitive Ly $\alpha$ instruments that aim to observe solar flares in the future.

The peak timings of the averaged profiles are also shown in Figure 4 as vertical dotted lines. For B-class flares, the average peak time coincides with the peak of the SXR emission for the summed events to within one GOES-15/EUVS-E time bin (10.24 s). For C-class flares, Ly $\alpha$ peaks slightly earlier than the X-ray peak (40.96 s; four time bins), while Mand X-class flares peak even earlier still (51.20 s and $71.68 \mathrm{~s}$, respectively). This somehow implies that Ly $\alpha$ emission from B-class flares may be thermally generated, while C-, M-, and X-class flares exhibit more nonthermal Ly $\alpha$ emission in accordance with the Neupert effect (Neupert, 1968). Although it is tempting to assume that this systematic change in peak timing is physically significant, great care must be taken in drawing conclusions from summed epoch analyses as individual events can sometimes skew the resulting profile.

\subsection{Center-to-Limb Variation}

As Ly $\alpha$ is optically thick (Woods et al., 2000), flares that occur closer to the solar limb are assumed to produce less of a response in Ly $\alpha$ than if they had occurred at disc center due to scattering by the increasing column mass along the line-of-sight. Milligan et al. (2020) showed that this was true for $31 \mathrm{X}$-class flares by normalising their $\operatorname{Ly} \alpha$ flux to their X-ray flux (which is optically thin) and showing that this ratio decreases with increasing heliocentric angle from disc center. This center-to-limb variation was also confirmed through a stereoscopic observation of an X-class limb event as viewed by GOES-15 from Earth that was observed simultaneously by the EUV Monitor on board the Mars Atmospheric and Volatile Evolution satellite (MAVEN/EUM; Eparvier et al., 2015), when Earth and Mars were around 90 degrees apart. After correcting for the Earth-Mars distance and light travel time, the flux detected by MAVEN (which observed the flare close to disc center) was greater than that detected by GOES.

Figure 5 shows averaged flare time profiles for the four GOES classes in increments of 10 degrees from $0-90^{\circ}$. In most cases, flares that occurred closer to disc center had a higher absolute flux value, and a more pronounced peak relative to the background around the time of the GOES SXR peak. This trend breaks down for X-class flares due to the diminishing number of events. To illustrate this point, Figure 6 shows the contrast value (peak flux divided by minimum flux) for each heliographic angle bin for B-, C-, and Mclass flares. Error bars were taken as the $1 \sigma$ value for each curve in Figure 5 divided by the square root of the number events in each bin (Kretzschmar et al., 2010). These data points were fitted with the commonly used CLV function:

$$
R=R_{C}\left(k+2(1-k)\left(\mu-\frac{\mu^{2}}{2}\right)\right),
$$




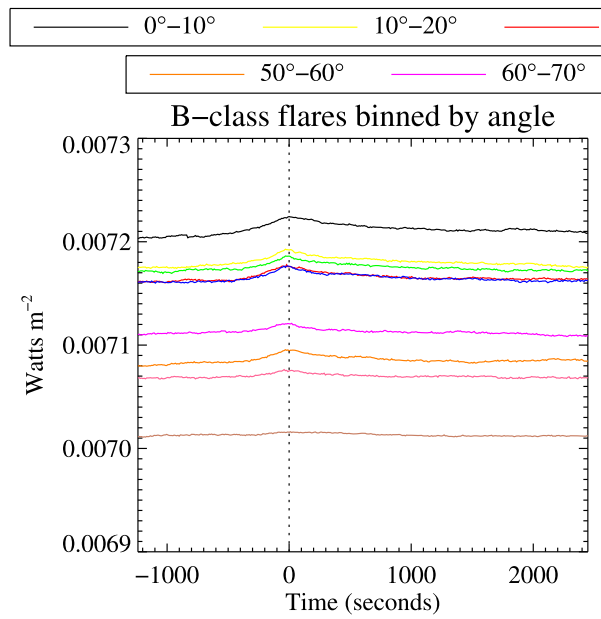

\begin{tabular}{c}
$20^{\circ}-30^{\circ}-30^{\circ}-40^{\circ} \longleftarrow 40^{\circ}-50^{\circ}$ \\
\hline $70^{\circ}-80^{\circ} \longleftarrow 80^{\circ}-90^{\circ}$
\end{tabular}
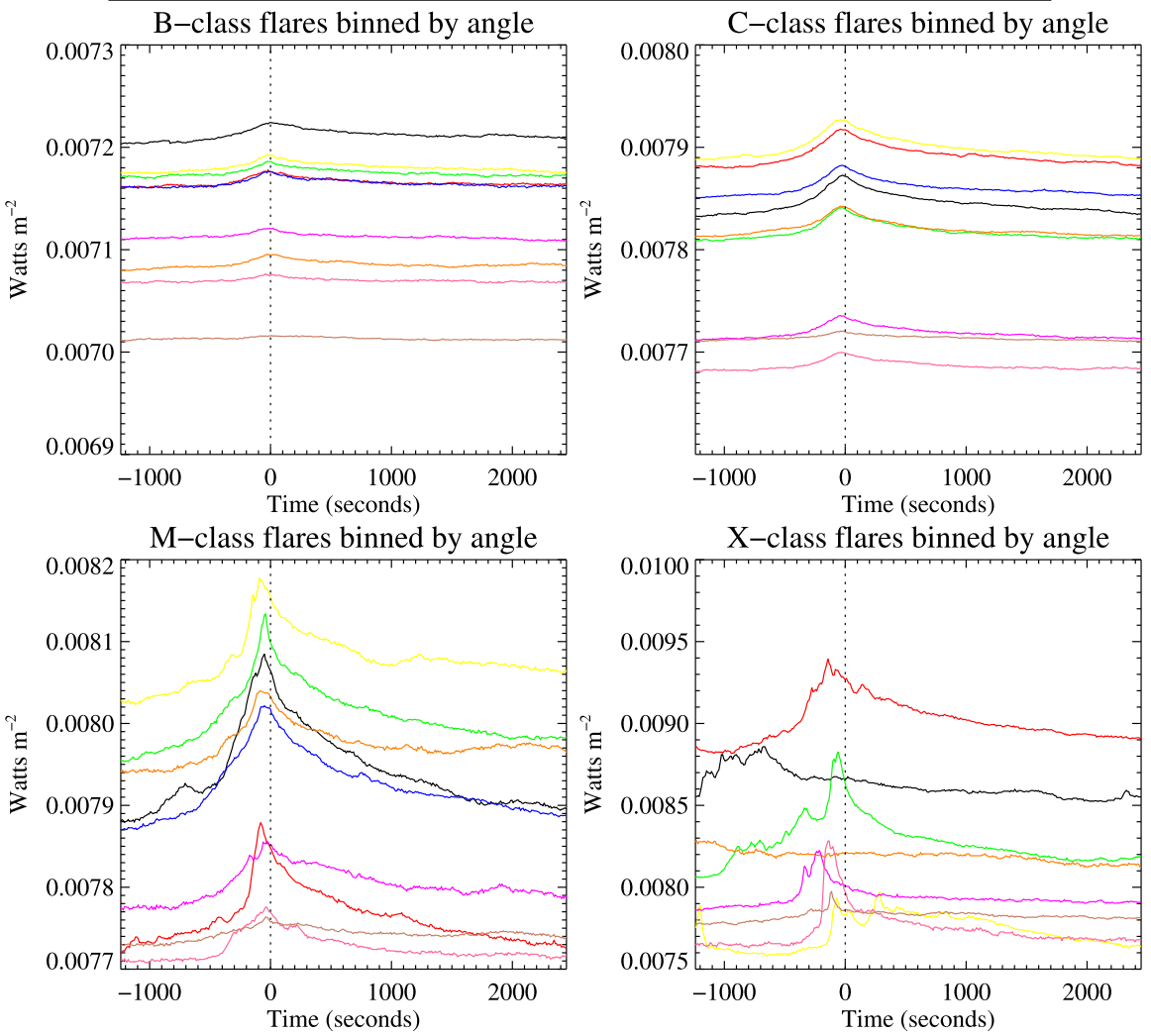

Figure 5 Plot of the Ly $\alpha$ time profiles for flares binned by heliographic angle in increments of 10 degrees for B-, C-, M-, and X-class flares. The vertical dashed line in each panel marks the time of the SXR peaks of all flares.

as derived by Brekke and Kjeldseth-Moe (1994) and also employed by Woods, Kopp, and Chamberlin (2006) and Milligan et al. (2020). $R$ is the intensity at a given angle, $R_{C}$ is the intensity ratio at disc center, $k$ is the limb variation relative to center, and $\mu=\cos (\theta)$. The values of $R_{C}$ for B-, C-, and M-class flares are 1.002, 1.004, and 1.021, respectively, while the corresponding $k$ values are $0.99,0.99$, and 0.97 . In agreement with Milligan et al. (2020), all flare classifications showed a smaller relative enhancement in Ly $\alpha$ emission for flares that occurred closer to the solar limb compared to those that occurred closer to disc center. While it would be more physically meaningful to normalize each curve to their respective GOES class (Woods, Kopp, and Chamberlin, 2006), the summed nature of the superposed-epoch analysis coupled with the difficulty associated with subtracting the solar background for smaller X-ray events, make this much more of a challenge, and is likely to return similar trends, albeit with much lower $k$-values. Note that Kretzschmar et al. (2010) and Kretzschmar (2011) did not include flares that occurred beyond $60^{\circ}$ of disc center in their superposed-epoch analysis on flares in the TSI, visible, EUV, and SXR emission as they assumed that limb flares would not produce a measurable response at these wavelengths in the chromosphere. 
Figure 6 Plot of the $\operatorname{Ly} \alpha$ contrast for each of the averaged lightcurves in Figure 5 (excluding X-class flares) as a function of heliographic angle. The curves fitted to each GOES class are given by Equation 1.
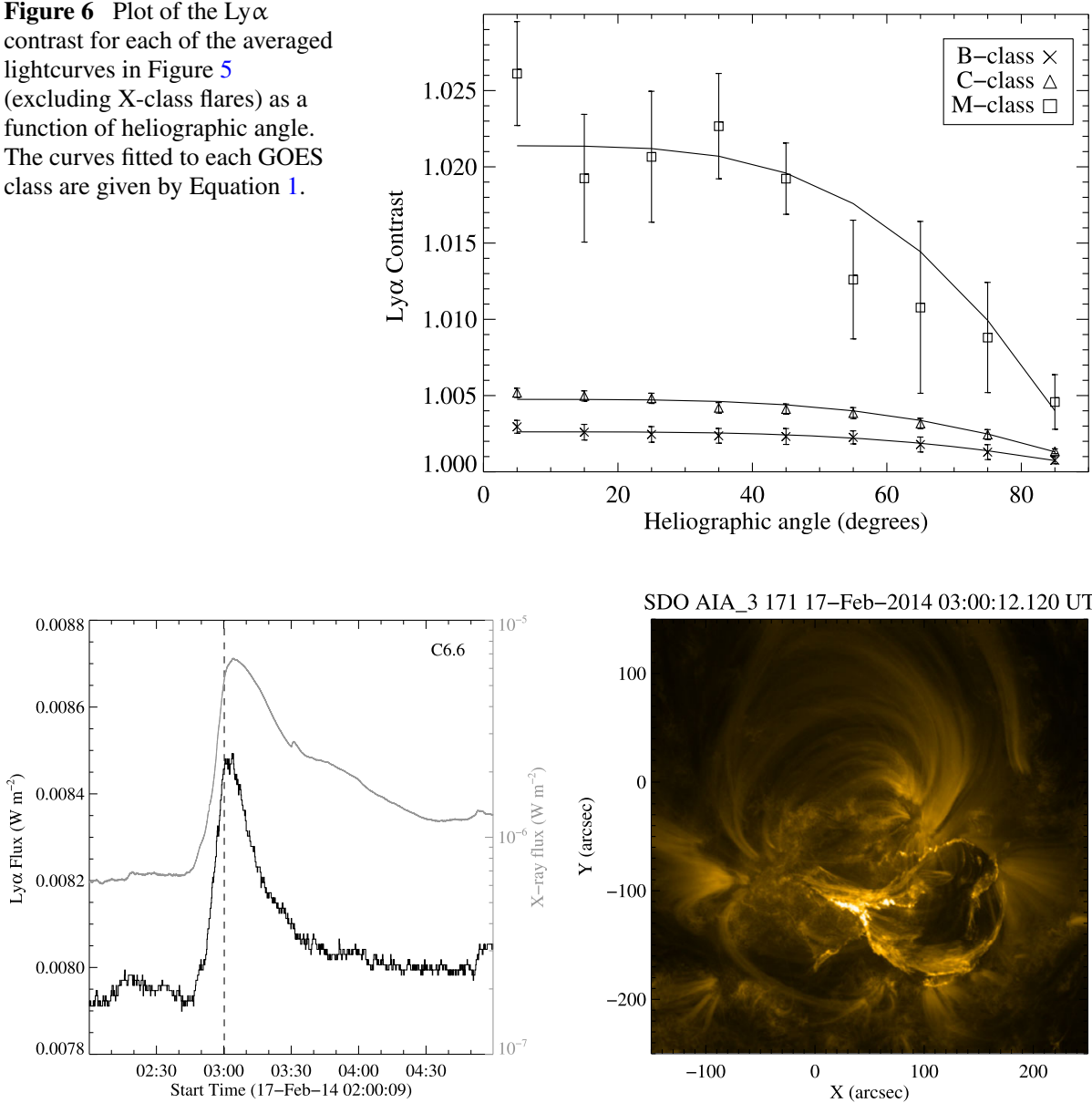

SDO AIA 3171 17-Feb-2014 03:00:12.120 UT

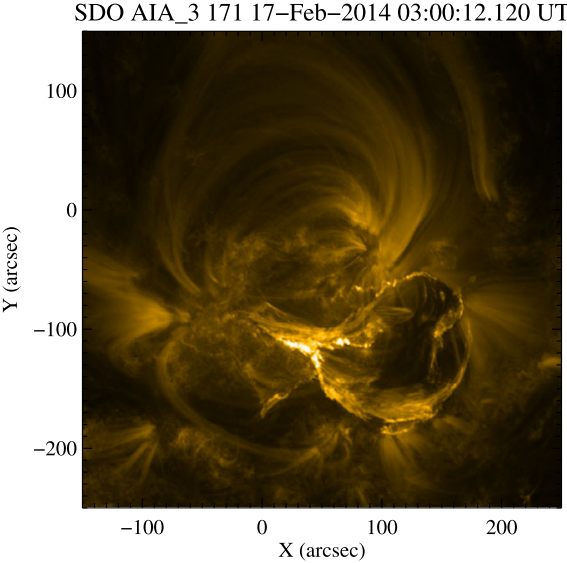

Figure 7 Left: Plot of Ly $\alpha$ (black) and X-ray (gray) time profiles for a C6.6 flare that occurred on 17 February 2014. The vertical dashed line denotes the time of the SDO/AIA image in the right hand panel. Right: SDO/AIA $171 \AA$ image taken around the time of peak Ly $\alpha$ emission showing the possible failed filament eruption that may be responsible for the unusually high Ly $\alpha$ flux.

\subsection{An Unusual C-class Flare}

While the vast majority of B- and C-class flares do not produce a Ly $\alpha$ signature above the bright solar background, one event in this study - a C6.6 flare that occurred on 17 February 2014 (SOL2014-02-17) — displayed a remarkable 7\% enhancement. Such a degree of contrast has hitherto only been associated with a few X-class flares (Woods et al., 2004; Milligan et al., 2020). Converting the flare excess in Ly $\alpha$ into units of energy for this event yielded a value of $\approx 10^{30} \mathrm{erg}$, which is comparable to that calculated for the 15 February 2011 X2.2 by Milligan et al. (2014) using Ly $\alpha$ data from the EUV Variability Experiment (EVE; Woods et al., 2012) on board the Solar Dynamics Observatory (SDO; Pesnell, Thompson, and Chamberlin, 2012). A plot of the Ly $\alpha$ and X-ray lightcurves for the event is shown in Figure 7, along with a $171 \AA$ image from the Atmospheric Imaging Assembly (AIA; Lemen et al., 2012), also on SDO, taken around the time of peak emission (vertical dashed line 
in left hand panel). Ordinarily, flare-related increases in Ly $\alpha$ (or indeed any inherent chromospheric emission) is primarily detected during the impulsive phase as the chromosphere is heated by precipitating particles from the corona, or late in the decay phase as hot flare plasma cools through instrumental passbands. Large increases in Ly $\alpha$ would therefore usually be attributed to a particularly energetic beam of electrons, and/or an unusually large footpoint area. Unfortunately, hard X-ray data from the Ramaty High Energy Solar Spectroscopic Imager (Lin et al., 2002) or Fermi (Meegan et al., 2009) were not available for this event, and so the parameters of the electron distribution that may have been responsible remains unknown. However, SDO/AIA data appear to show a failed filament eruption in all EUV channels (only $171 \AA$ is shown in Figure 7). No associated coronal mass ejection could be seen in coronagraph images around the time and location of the flare. The Ly $\alpha$ emission from this event appears to correlate well with the main X-ray phase suggesting that the it could have been emanating from the corona as heating took place within the large volume of the filament. A similar conclusion was reached by Rubio da Costa et al. (2009) who reported co-spatial Ly $\alpha$ (from the Transition Region and Coronal Explorer; Handy et al., 1999) and $\mathrm{X}$-ray emission during a filament eruption.

\section{Conclusions}

This article presents a study of 8579 solar flares observed in the Ly $\alpha$ line of neutral hydrogen. As weaker flares do not readily produce a Ly $\alpha$ signature above the intense solar background at this wavelength, a superposed-epoch analysis was carried out on 3123 B-class flares and 4972 C-class flares. On average, these flares were found to exhibit a $0.1-0.3 \%$ enhancement. For completion and comparison, $453 \mathrm{M}$ - and $31 \mathrm{X}$-flares were also coadded, and average increases of $1-4 \%$ were measured. For each GOES classification, flares were also summed according to their heliographic location revealing that in all cases, flares closer to disc center displayed a greater enhancement on average than those that occurred closer to the solar limb due to the opacity of $\operatorname{Ly} \alpha$, or possible foreshortening of the flare ribbons. A C-class flare that exhibited an abnormally high contrast of $7 \%$ (equating to $10^{30} \mathrm{erg}$ ) was also presented.

While GOES/EUVS was designed as an EUV irradiance monitor, the results presented here show just how stable the data are and how they can be utilized for detailed scientific analysis, in particular for large scale statistical studies. Although the sensitivity of GOES15/EUVS-E might be limited to larger flares, the new Ly $\alpha$ irradiance instruments that are part of the Extreme Ultraviolet and X-ray Irradiance Sensor (EXIS; Eparvier et al., 2009; Chamberlin et al., 2009) suite on the newly launched GOES-R series of spacecraft (GOES16 and GOES-17 were launched in February 2017 and June 2018, respectively, with GOES18 and GOES-19 to follow) will have a greater dynamic range, as well as will provide pseudo line profiles by sampling the Ly $\alpha$ profile in five wavelength pixels rather than as broadband measurements. Similarly, the imaging capability of the EUV Imager (EUI; Schühle et al., 2011; Rochus et al., 2020) on board Solar Orbiter will be able to spatially resolve flares in Ly $\alpha$ for the first time. The findings presented illustrate that even minor flares can produce small, but perceptible changes in the solar Ly $\alpha$ irradiance, and should therefore serve as a baseline for the advent of new Ly $\alpha$ flare observations and advanced numerical simulations that will become available during Solar Cycle 25.

Acknowledgements The author would like to thank Science and Technologies Facilities Council (UK) for the award of an Ernest Rutherford Fellowship (ST/N004981/2), and Hugh Hudson, Mihalis Mathioudakis, Graham Kerr, and Shaun McLaughlin for feedback on an early draft. The GOES data used in this study 
are freely and publicly available from the instrument websites (GOES/EUVS: https://satdat.ngdc.noaa.gov/ sem/goes/data/euvs/GOES_v4/G15/; GOES/XRS: https://www.ngdc.noaa.gov/stp/satellite/goes/dataaccess. html).

\section{Disclosure of Potential Conflicts of Interest The author declares that he has no conflicts of interest.}

Publisher's Note Springer Nature remains neutral with regard to jurisdictional claims in published maps and institutional affiliations.

Open Access This article is licensed under a Creative Commons Attribution 4.0 International License, which permits use, sharing, adaptation, distribution and reproduction in any medium or format, as long as you give appropriate credit to the original author(s) and the source, provide a link to the Creative Commons licence, and indicate if changes were made. The images or other third party material in this article are included in the article's Creative Commons licence, unless indicated otherwise in a credit line to the material. If material is not included in the article's Creative Commons licence and your intended use is not permitted by statutory regulation or exceeds the permitted use, you will need to obtain permission directly from the copyright holder. To view a copy of this licence, visit http://creativecommons.org/licenses/by/4.0/.

\section{References}

Baliukin, I.I., Bertaux, J.-L., Quémerais, E., Izmodenov, V.V., Schmidt, W.: 2019, SWAN/SOHO Lyman- $\alpha$ mapping: the hydrogen geocorona extends well beyond the Moon. J. Geophys. Res. Space Phys. 124, 861. DOI. ADS.

Brekke, P., Kjeldseth-Moe, O.: 1994, The solar UV continuum 1440-1680 A and its center-to-limb variation. Solar Phys. 150, 19. DOI. ADS.

Brekke, P., Rottman, G.J., Fontenla, J., Judge, P.G.: 1996, The ultraviolet spectrum of a 3 B class flare observed with SOLSTICE. Astrophys. J. 468, 418. DOI. ADS.

Canfield, R.C., van Hoosier, M.E.: 1980, Observed L-alpha profiles for two solar flares - 14:12 UT 15 June, 1973 and 23:16 UT 21 January, 1974. Solar Phys. 67, 339. DOI. ADS.

Chamberlin, P.C., Woods, T.N., Eparvier, F.G., Jones, A.R.: 2009, Next Generation X-Ray Sensor (XRS) for the NOAA GOES-R Satellite Series, SPIE Conf. Ser. 7438, 743802. DOI. ADS.

Chubb, T.A., Friedman, H., Kreplin, R.W., Kupperian, J.E Jr.: 1957, Lyman alpha and X-ray emissions during a small solar flare. J. Geophys. Res. 62, 389. DOI. ADS.

Dominique, M., Zhukov, A.N., Heinzel, P., Dammasch, I.E., Wauters, L., Dolla, L., Shestov, S., Kretzschmar, M., Machol, J., Lapenta, G., Schmutz, W.: 2018, First detection of solar flare emission in mid-ultraviolet Blamer continuum. Astrophys. J. Lett. 867, L24. DOI. ADS.

Eparvier, F.G., Crotser, D., Jones, A.R., McClintock, W.E., Snow, M., Woods, T.N.: 2009, The Extreme Ultraviolet Sensor (EUVS) for GOES-R, SPIE Conf. Ser. 7438, 743804. DOI. ADS.

Eparvier, F.G., Chamberlin, P.C., Woods, T.N., Thiemann, E.M.B.: 2015, The solar extreme ultraviolet monitor for MAVEN. Space Sci. Rev. 195, 293. DOI. ADS.

Handy, B.N., Acton, L.W., Kankelborg, C.C., Wolfson, C.J., Akin, D.J., Bruner, M.E., Caravalho, R., Catura, R.C., Chevalier, R., Duncan, D.W., Edwards, C.G., Feinstein, C.N., Freeland, S.L., Friedlaender, F.M., Hoffmann, C.H., Hurlburt, N.E., Jurcevich, B.K., Katz, N.L., Kelly, G.A., Lemen, J.R., Levay, M., Lindgren, R.W., Mathur, D.P., Meyer, S.B., Morrison, S.J., Morrison, M.D., Nightingale, R.W., Pope, T.P., Rehse, R.A., Schrijver, C.J., Shine, R.A., Shing, L., Strong, K.T., Tarbell, T.D., Title, A.M., Torgerson, D.D., Golub, L., Bookbinder, J.A., Caldwell, D., Cheimets, P.N., Davis, W.N., Deluca, E.E., McMullen, R.A., Warren, H.P., Amato, D., Fisher, R., Maldonado, H., Parkinson, C.: 1999, The transition region and coronal explorer. Solar Phys. 187, 229. DOI. ADS.

Hanser, F.A., Sellers, F.B.: 1996, Design and calibration of the GOES-8 solar x-ray sensor: the XRS. In: Washwell, E.R. (ed.) GOES-8 and Beyond, Proc. SPIE 2812, 344. DOI. ADS.

Kretzschmar, M.: 2011, The Sun as a star: observations of white-light flares. Astron. Astrophys. 530, A84. DOI. ADS.

Kretzschmar, M.: 2015, Temperature dependence of the flare fluence scaling exponent. Solar Phys. $290,3593$. DOI. ADS.

Kretzschmar, M., Dominique, M., Dammasch, I.E.: 2013, Sun-as-a-star observation of flares in Lyman $\alpha$ by the PROBA2/LYRA radiometer. Solar Phys. 286, 221. DOI. ADS.

Kretzschmar, M., de Wit, T.D., Schmutz, W., Mekaoui, S., Hochedez, J.-F., Dewitte, S.: 2010, The effect of flares on total solar irradiance. Nat. Phys. 6, 690. DOI. ADS. 
Lean, J.L.: 1985, Calculations of Lyman alpha absorption in the mesosphere. In: Zerefos, C.S., Ghazi, A. (eds.) Atmospheric Ozone, 697. Springer, Dordrecht. ADS.

Lemen, J.R., Title, A.M., Akin, D.J., Boerner, P.F., Chou, C., Drake, J.F., Duncan, D.W., Edwards, C.G., Friedlaender, F.M., Heyman, G.F., Hurlburt, N.E., Katz, N.L., Kushner, G.D., Levay, M., Lindgren, R.W., Mathur, D.P., McFeaters, E.L., Mitchell, S., Rehse, R.A., Schrijver, C.J., Springer, L.A., Stern, R.A., Tarbell, T.D., Wuelser, J.-P., Wolfson, C.J., Yanari, C., Bookbinder, J.A., Cheimets, P.N., Caldwell, D., Deluca, E.E., Gates, R., Golub, L., Park, S., Podgorski, W.A., Bush, R.I., Scherrer, P.H., Gummin, M.A., Smith, P., Auker, G., Jerram, P., Pool, P., Soufli, R., Windt, D.L., Beardsley, S., Clapp, M., Lang, J., Waltham, N.: 2012, The Atmospheric Imaging Assembly (AIA) on the Solar Dynamics Observatory (SDO). Solar Phys. 275, 17. DOI. ADS.

Lin, R.P., Dennis, B.R., Hurford, G.J., Smith, D.M., Zehnder, A., Harvey, P.R., Curtis, D.W., Pankow, D., Turin, P., Bester, M., Csillaghy, A., Lewis, M., Madden, N., van Beek, H.F., Appleby, M., Raudorf, T., McTiernan, J., Ramaty, R., Schmahl, E., Schwartz, R., Krucker, S., Abiad, R., Quinn, T., Berg, P., Hashii, M., Sterling, R., Jackson, R., Pratt, R., Campbell, R.D., Malone, D., Landis, D., BarringtonLeigh, C.P., Slassi-Sennou, S., Cork, C., Clark, D., Amato, D., Orwig, L., Boyle, R., Banks, I.S., Shirey, K., Tolbert, A.K., Zarro, D., Snow, F., Thomsen, K., Henneck, R., McHedlishvili, A., Ming, P., Fivian, M., Jordan, J., Wanner, R., Crubb, J., Preble, J., Matranga, M., Benz, A., Hudson, H., Canfield, R.C., Holman, G.D., Crannell, C., Kosugi, T., Emslie, A.G., Vilmer, N., Brown, J.C., Johns-Krull, C., Aschwanden, M., Metcalf, T., Conway, A.: 2002, The Reuven Ramaty High-Energy Solar Spectroscopic Imager (RHESSI). Solar Phys. 210, 3. DOI. ADS.

McClintock, W.E., Rottman, G.J., Woods, T.N.: 2005, Solar-stellar irradiance comparison experiment II (Solstice II): instrument concept and design. Solar Phys. 230, 225. DOI. ADS.

Meegan, C., Lichti, G., Bhat, P.N., Bissaldi, E., Briggs, M.S., Connaughton, V., Diehl, R., Fishman, G., Greiner, J., Hoover, A.S., van der Horst, A.e.J., von Kienlin, A., Kippen, R.M., Kouveliotou, C., McBreen, S., Paciesas, W.S., Preece, R., Steinle, H., Wallace, M.S., Wilson, R.B., Wilson-Hodge, C.: 2009, The Fermi gamma-ray burst monitor. Astrophys. J. 702, 791. DOI. ADS.

Meier, R.R., Prinz, D.K.: 1970, Absorption of the solar Lyman alpha line by geocoronal atomic hydrogen. $J$. Geophys. Res. 75, 6969. DOI. ADS.

Milligan, R.O., Chamberlin, P.C.: 2016, Anomalous temporal behaviour of broadband Ly $\alpha$ observations during solar flares from SDO/EVE. Astron. Astrophys. 587, A123. DOI. ADS.

Milligan, R.O., Ireland, J.: 2018, On the performance of multi-instrument solar flare observations during solar cycle 24. Solar Phys. 293, 18. DOI. ADS.

Milligan, R.O., Kerr, G.S., Dennis, B.R., Hudson, H.S., Fletcher, L., Allred, J.C., Chamberlin, P.C., Ireland, J., Mathioudakis, M., Keenan, F.P.: 2014, The radiated energy budget of chromospheric plasma in a major solar flare deduced from multi-wavelength observations. Astrophys. J. 793, 70. DOI. ADS.

Milligan, R.O., Fleck, B., Ireland, J., Fletcher, L., Dennis, B.R.: 2017, Detection of three-minute oscillations in full-disk Ly $\alpha$ emission during a solar flare. Astrophys. J. Lett. 848, L8. DOI. ADS.

Milligan, R.O., Hudson, H.S., Chamberlin, P.C., Hannah, I.G., Hayes, L.A.: 2020, Lyman-alpha variability during solar flares over solar cycle 24 using GOES-15/EUVS-E. Space Weather 18, e2331. DOI. ADS.

Neupert, W.M.: 1968, Comparison of solar X-ray line emission with microwave emission during flares. Astrophys. J. Lett. 153, L59. DOI. ADS.

Pesnell, W.D., Thompson, B.J., Chamberlin, P.C.: 2012, The Solar Dynamics Observatory (SDO). Solar Phys. 275, 3. DOI. ADS.

Raulin, J.-P., Trottet, G., Kretzschmar, M., Macotela, E.L., Pacini, A.r., Bertoni, F.C.P., Dammasch, I.E.: 2013, Response of the low ionosphere to X-ray and Lyman- $\alpha$ solar flare emissions. J. Geophys. Res. Space Phys. 118, 570. DOI. ADS.

Rochus, P., Auchère, F., Berghmans, D., Harra, L., Schmutz, W., Schühle, U., Addison, P., Appourchaux, T., Aznar Cuadrado, R., Baker, D., Barbay, J., Bates, D., BenMoussa, A., Bergmann, M., Beurthe, C., Borgo, B., Bonte, K., Bouzit, M., Bradley, L., Büchel, V., Buchlin, E., Büchner, J., Cabé, F., Cadiergues, L., Chaigneau, M., Chares, B., Choque Cortez, C., Coker, P., Condamin, M., Coumar, S., Curdt, W., Cutler, J., Davies, D., Davison, G., Defise, J.-M., Del Zanna, G., Delmotte, F., Delouille, V., Dolla, L., Dumesnil, C., Dürig, F., Enge, R., François, S., Fourmond, J.-J., Gillis, J.-M., Giordanengo, B., Gissot, S., Green, L.M., Guerreiro, N., Guilbaud, A., Gyo, M., Haberreiter, M., Hafiz, A., Hailey, M., Halain, J.-P., Hansotte, J., Hecquet, C., Heerlein, K., Hellin, M.-L., Hemsley, S., Hermans, A., Hervier, V., Hochedez, J.-F., Houbrechts, Y., Ihsan, K., Jacques, L., Jérôme, A., Jones, J., Kahle, M., Kennedy, T., Klaproth, M., Kolleck, M., Koller, S., Kotsialos, E., Kraaikamp, E., Langer, P., Lawrenson, A., Le Clech', J.-C., Lenaerts, C., Liebecq, S., Linder, D., Long, D.M., Mampaey, B., Markiewicz-Innes, D., Marquet, B., Marsch, E., Matthews, S., Mazy, E., Mazzoli, A., Meining, S., Meltchakov, E., Mercier, R., Meyer, S., Monecke, M., Monfort, F., Morinaud, G., Moron, F., Mountney, L., Müller, R., Nicula, B., Parenti, S., Peter, H., Pfiffner, D., Philippon, A., Phillips, I., Plesseria, J.-Y., Pylyser, E., Rabecki, F., Ravet-Krill, M.-F., Rebellato, J., Renotte, E., Rodriguez, L., Roose, S., Rosin, J., Rossi, L., Roth, P., 
Rouesnel, F., Roulliay, M., Rousseau, A., Ruane, K., Scanlan, J., Schlatter, P., Seaton, D.B., Silliman, K., Smit, S., Smith, P.J., Solanki, S.K., Spescha, M., Spencer, A., Stegen, K., Stockman, Y., Szwec, N., Tamiatto, C., Tandy, J., Teriaca, L., Theobald, C., Tychon, I., van Driel-Gesztelyi, L., Verbeeck, C., Vial, J.-C., Werner, S., West, M.J., Westwood, D., Wiegelmann, T., Willis, G., Winter, B., Zerr, A., Zhang, X., Zhukov, A.N.: 2020, The Solar Orbiter EUI instrument: the extreme ultraviolet imager. Astron. Astrophys. 642, A8. DOI. ADS.

Rubio da Costa, F., Fletcher, L., Labrosse, N., Zuccarello, F.: 2009, Observations of a solar flare and filament eruption in Lyman $\alpha$ and X-rays. Astron. Astrophys. 507, 1005. DOI. ADS.

Schühle, U., Halain, J.-P., Meining, S., Teriaca, L.: 2011, The Lyman-alpha telescope of the extreme ultraviolet imager on Solar Orbiter. In: Fineschi, S., Fennelly, J. (eds.) Solar Phys. and Space Weather Instrumentation IV, SPIE Conf. Ser. 8148, 81480K. DOI. ADS.

Viereck, R., Hanser, F., Wise, J., Guha, S., Jones, A., McMullin, D., Plunket, S., Strickland, D., Evans, S.: 2007, Solar extreme ultraviolet irradiance observations from GOES: design characteristics and initial performance. In: Fineschi, S., Viereck, R.A. (eds.) Solar Phys. and Space Weather Instrumentation II, SPIE Conf. Ser. 6689, 66890K. DOI. ADS.

Woods, T.N., Kopp, G., Chamberlin, P.C.: 2006, Contributions of the solar ultraviolet irradiance to the total solar irradiance during large flares. J. Geophys. Res. Space Phys. 111, A10S14. DOI. ADS.

Woods, T.N., Rottman, G.J., White, O.R., Fontenla, J., Avrett, E.H.: 1995, The solar LY alpha line profile. Astrophys. J. 442, 898. DOI. ADS.

Woods, T.N., Tobiska, W.K., Rottman, G.J., Worden, J.R.: 2000, Improved solar Lyman $\alpha$ irradiance modeling from 1947 through 1999 based on UARS observations. J. Geophys. Res. 105, 27195. DOI. ADS.

Woods, T.N., Eparvier, F.G., Fontenla, J., Harder, J., Kopp, G., McClintock, W.E., Rottman, G., Smiley, B., Snow, M.: 2004, Solar irradiance variability during the October 2003 solar storm period. Geophys. Res. Lett. 31, L10802. DOI. ADS.

Woods, T.N., Chamberlin, P.C., Harder, J.W., Hock, R.A., Snow, M., Eparvier, F.G., Fontenla, J., McClintock, W.E., Richard, E.C.: 2009, Solar irradiance reference spectra (SIRS) for the 2008 whole heliosphere interval (WHI). Geophys. Res. Lett. 36, L01101. DOI. ADS.

Woods, T.N., Eparvier, F.G., Hock, R., Jones, A.R., Woodraska, D., Judge, D., Didkovsky, L., Lean, J., Mariska, J., Warren, H., McMullin, D., Chamberlin, P., Berthiaume, G., Bailey, S., Fuller-Rowell, T., Sojka, J., Tobiska, W.K., Viereck, R.: 2012, Extreme Ultraviolet Variability Experiment (EVE) on the Solar Dynamics Observatory (SDO): overview of science objectives, instrument design, data products, and model developments. Solar Phys. 275, 115. DOI. ADS. 